\title{
Erratum to: "Comments on another hybrid conjugate gradient algorithm for unconstrained optimization by Andrei"
}

\section{Zhifeng Dai ${ }^{1} \cdot$ Fenghua Wen $^{2}$}

Published online: 8 September 2015

(C) Springer Science+Business Media New York 2015

\section{Erratum to: Numer Algor (2015) 69:337-341 \\ DOI 10.1007/s11075-014-9899-8}

We would like to correct some details of the above paper. According to References [1] and formula (2.8) in the above paper, we give some corrections about the above paper. More precisely,

- The formula (2.9) in Theorem 2.1 should be

$$
g_{k+1}^{T} d_{k+1} \leq-\delta\left\|g_{k+1}\right\|^{2} .
$$

- Formula (3.1) should be

$$
g_{k}^{T} s_{k} \leq-\delta\left\|g_{k}\right\|^{2}
$$

- Formula (3.2) should be

$$
g_{k}^{T} d_{k} \leq-\delta\left\|g_{k}\right\|^{2}
$$

- Formula (3.3) should be

$$
g_{k}^{T} s_{k} \leq-\alpha_{k} \delta\left\|g_{k}\right\|^{2} .
$$

- Formula (3.9) should be

$$
g_{k}^{T} s_{k} \leq-\alpha_{k} \delta\left\|g_{k}\right\|^{2} \text {. }
$$

The online version of the original article can be found at http://dx.doi.org/10.1007/s11075-014-9899-8.

Fenghua Wen

wfh@amss.ac.cn

Zhifeng Dai

zhifengdai823@163.com

1 College of Mathematics and Computational Science, Changsha University of Science and Technology, Changsha 410114, China

2 College of Business, Central South University, Hunan, 410083, China 
- Formula (3.10) should be

$$
y_{k}^{T} s_{k} \geq \alpha_{k}(1-\sigma) \delta\left\|g_{k}\right\|^{2} .
$$

Everything else in the paper remains unchanged. The authors would like to apologise for any inconvenience caused.

Acknowledgments This work is supported by the NSF of China granted 11301041, 71371195, 71431008, Project funded by China Postdoctoral Science Foundation(2015T80893), Natural Science Foundation of Hunan Province(2015JJ3015).

\section{References}

1. Andrei, N.: Another hybrid conjugate gradient algorithm for unconstrained optimization. Numer. Algor. 47, 143-156 (2008) 